\title{
Achados da ressonância magnética e desfechos neurológicos em recém-nascidos com Encefalopatia Hipóxico-Isquêmica: revisão integrativa
}

Findings of magnetic resonance and neurological outcome in newborns with Hypoxic Ischemic Encephalopathy: integrative review

Hallazgos de resonancia magnética y resultados neurológicos en recién nacidos con Encefalopatía Isquémica Hipóxica: revisión integrativa

Recebido: 23/08/2021 | Revisado: 28/08/2021 | Aceito: 31/08/2021 | Publicado: 04/09/2021

\author{
Beatriz Carvalho Ferreira \\ ORCID: https://orcid.org/0000-0002-5202-283X \\ Universidade Federal de Sergipe, Brasil \\ E-mail: beatrizcarv.ferreira@gmail.com \\ Alice Cardoso \\ ORCID: https://orcid.org/0000-0001-7835-3306 \\ Universidade Federal de Sergipe, Brasil \\ E-mail: ali_ce_cardoso@hotmail.com \\ Luana Teles de Resende \\ ORCID: https://orcid.org/0000-0002-6223-9186 \\ Universidade Federal de Sergipe, Brasil \\ E-mail: lua.teles.resende@gmail.com \\ Aglaé da Silva Araújo Andrade \\ ORCID: https://orcid.org/0000-0003-1832-7899 \\ Universidade Federal de Sergipe, Brasil \\ E-mail: a3glae@yahoo.com.br \\ Halley Ferraro Oliveira \\ ORCID: https://orcid.org/0000-0003-0123-7395 \\ Universidade Federal de Sergipe, Brasil \\ E-mail: Halleyoliveira62@gmail.com \\ Andreia Centenaro Vaez \\ ORCID: https://orcid.org/0000-0002-1503-0785 \\ Universidade Federal de Sergipe, Brasil \\ E-mail: Andreiacentenarovaez@gmail.com
}

\begin{abstract}
Resumo
O objetivo deste estudo foi analisar os principais achados nos exames de neuroimagem e os desfechos no neurodesenvolvimento em crianças com encefalopatia hipóxico-isquêmica. Trata-se de uma revisão integrativa realizada nas bases CINAHL e PubMed, com os seguintes critérios de inclusão: estudos de coorte, ensaios clínicos, publicações dos últimos 5 anos, idiomas em português, inglês e espanhol, lactente: nascimento-23 meses, lactente: 1-23 meses, humanos, diagnóstico por ressonância magnética, tomografia e eletroencefalograma. Dos 2.248 artigos que foram identificados, 11 foram selecionados para compor o estudo. Constatou-se por meio dos exames de imagem a prevalência de lesões nos Gânglios da Base e Tálamo e na Substância Branca. Os principais resultados neurológicos identificados caracterizaramse como atraso motor, cognitivo e de linguagem. Evidenciou-se uma maior frequência de lesões em Gânglios da Base e Tálamo e Substância Branca, por meio da ressonância magnética. Em relação ao desenvolvimento infantil, os principais desfechos identificados foram atraso motor, cognitivo e de linguagem. Este estudo aponta como limitações o acesso limitado às técnicas de neuroimagem em países em desenvolvimento, bem como a falta de instrumentos padronizados para avaliação do desenvolvimento infantil.

Palavras-chave: Asfixia neonatal; Encefalopatia hipóxica-isquêmica; Recém-nascido; Diagnóstico por imagem; Transtornos do neurodesenvolvimento.

\footnotetext{
Abstract

The aim of the study was to analyze the main findings in neuroimaging exams and neurodevelopmental outcomes in children with hypoxic-ischemic encephalopathy. This is an integrative review in which searches were performed on the CINAHL and PubMed databases, with the following inclusion criteria: cohort studies, clinical trials, publications from the last 5 years in the languages of Portuguese, English and Spanish. Infant: birth-23 months, Infant: 1-23 months, human, diagnosis by magnetic resonance, tomography and electroencephalogram. Of the 2,248 articles that were identified, 11 were selected to compose the study. Imaging tests showed the prevalence of lesions in the basal ganglia and
} 
thalamus and in white matter. The main neurological results identified were characterized as motor, cognitive and language delay. There was a higher frequency of lesions in Basal Ganglia, Thalamus and in the White Matter was discovered by means of magnetic resonance imaging. Regarding child development, the main outcomes identified were motor, cognitive and language delay. This study points out the disadvantages caused by the limited access to neuroimaging techniques in developing countries, as well as the lack of standardized instruments for assessing child development.

Keywords: Asphyxia neonatorum; Hypoxia-ischemia, brain; Infant, newborn; Diagnostic imaging; Neurodevelopmental disorders.

\section{Resumen}

El objetivo del estudio fue analizar los principales hallazgos en los exámenes de neuroimagen y los resultados del neurodesarrollo en niños con encefalopatía hipóxico-isquémica. Se trata de una revisión integradora en la que se realizaron búsquedas en las bases de datos CINAHL y PubMed, con los siguientes criterios de inclusión: estudios de cohortes, ensayos clínicos, publicaciones de los últimos 5 años, idiomas en portugués, inglés y español, infantil: nacimiento23 meses, lactante: 1-23 meses, humanos, diagnóstico por resonancia magnética, tomografía computarizada y electroencefalograma. De los 2.248 artículos identificados, se seleccionaron 11 para componer el estudio. La prevalencia de lesiones en los Ganglios Basales, Tálamo y en la Sustancia Blanca se verificó mediante exámenes de imagen. Los principales resultados neurológicos identificados se caracterizaron como retraso motor, cognitivo y del lenguaje. Hubo una mayor frecuencia de lesiones en los Ganglios Basales, Tálamo y Sustancia Blanca, mediante resonancia magnética. En cuanto al desarrollo infantil, los principales resultados identificados fueron el retraso motor, cognitivo y del lenguaje. Este estudio señala como limitaciones el acceso limitado a las técnicas de neuroimagen en los países en desarrollo, así como la falta de instrumentos estandarizados para la evaluación del desarrollo infantil.

Palabras claves: Asfixia neonatal; Hipoxia-isquemia encefálica; Recién nacido; Diagnóstico por imagen; Trastornos del neurodesarrollo.

\section{Introdução}

A Asfixia Perinatal (AP) é uma das principais causas da mortalidade neonatal no mundo (World Health Organization [WHO], 2021), caracterizada pela falta de oxigênio ou diminuição na perfusão tecidual do feto ou neonato. A etiologia pode estar relacionada à suspensão do fluxo sanguíneo pelo cordão umbilical, troca deficiente de gases pela placenta, hipotensão materna, feto comprometido que não suporta o trabalho de parto ou imaturidade pulmonar após o nascimento (Sociedade Brasileira de Pediatria [SBP], 2017).

Apesar da diminuição das taxas de mortalidade neonatal, a AP destaca-se por ser a segunda causa de morte em recémnascidos, representando $24,11 \%$ dos óbitos em âmbito mundial (WHO, 2021). Em esfera nacional, destaca-se por apresentar elevação das suas taxas, passando da quarta causa de morte em 1990 para a terceira em 2015 (França et al., 2017). Ressalta-se que os dados relacionados ao impacto da asfixia na morbimortalidade podem ser desvalorizados por razões como a falta de informações, preenchimento errôneo da declaração de óbito e a subnotificação (Rêgo, Vilela \& De Oliveira, 2018).

A Encefalopatia Hipóxico-Isquêmica (EHI) é a consequência da AP mais descrita na literatura, por ser caracterizada como o desfecho mais grave desse evento perinatal (Gunn \& Thoresen, 2019; SBP, 2017). As manifestações clínicas da EHI ocorrem por condições que indicam distúrbio neurológico e devem ser avaliadas criteriosamente (SBP, 2020). Dentre elas, a mais frequente é a crise convulsiva, que pode estar associada à depressão neurológica e, menos frequentemente, à dispneia. Ainda pode ocorrer diminuição do nível de consciência, assim como cianose, hipotonia e parada cardiorrespiratória (Abreu, 2018). As principais sequelas decorrentes da EHI são os atrasos ou desabilidades no desenvolvimento motor, cognitivo e de linguagem, bem como paralisia cerebral, cegueira, deficiência auditiva e morte (Ballot et al., 2020; Binkowski \& Weinmann, 2015).

A avaliação clínica detalhada é uma ferramenta de baixo custo e eficaz, auxilia na detecção de anormalidades do desenvolvimento e identificação da gravidade dos déficits neurológicos (Perez, Golombek, \& Sola, 2017). A partir da avaliação, podem ser classificadas de acordo com o sistema de estadiamento de Sarnat e Sarnat (1976), que avalia o grau de encefalopatia e classifica em três estágios: leve (estágio I), moderado (estágio II) e grave (estágio III), por meio dos seguintes critérios: nível de consciência, atividade espontânea, tônus muscular, reflexos, presença de disfunção autonômica e atividade elétrica do cérebro, 
através do eletroencefalograma (EEG).

Atualmente, associado à avaliação clínica, o uso da neuroimagem tem se tornado cada vez mais presente na prática clínica, visto que os avanços tecnológicos possibilitaram uma avaliação mais detalhada das alterações estruturais e funcionais do cérebro (Azhari et al., 2020; Ferreira, 2019). Nesse cenário, faz-se presente uma série de modalidades da neuroimagem, as quais se destacam o eletroencefalograma (EEG), magnetoencefalografia (MEG), a espectroscopia funcional de infravermelho próximo (fNIRS) e a ressonância magnética (Azhari et al., 2020). Esta última torna-se cada vez mais relevante na avaliação das crianças com encefalopatia (Charon et al., 2016).

Diante desse contexto, o presente estudo tem como objetivo analisar os principais achados nos exames de neuroimagem e os desfechos no neurodesenvolvimento em crianças com EHI. O conhecimento dessas informações pode auxiliar no prognóstico e acompanhamento especializado dessas crianças, tendo em vista a intervenção com estimulação precoce e a diminuição de possíveis desfechos neurológicos mais graves.

\section{Metodologia}

O presente estudo é uma revisão integrativa, realizada em seis etapas: (1) identificação do tema e seleção da questão de pesquisa; (2) estabelecimento dos critérios de inclusão e exclusão; (3) identificação dos estudos pré-selecionados e selecionados; (4) categorização dos estudos selecionados; (5) análise e interpretação dos resultados; e (6) apresentação da revisão/síntese do conhecimento (Botelho, Cunha, \& Macedo, 2011).

Para elaboração da questão de pesquisa, adotou-se a estratégia PICOT (Stillwell, Fineout-Overholt, Melnyk, \& Williamson, 2010), a qual leva em consideração a população alvo (P), intervenção (I), comparação de intervenção ou grupo (C), resultados (O) e tempo (T). Assim, a partir dessa estratégia, definiu-se: crianças com Encefalopatia Hipóxico-Isquêmica (P), avaliação por exames de imagem (I), desfechos neurológicos (O), acompanhamento até os dois anos de idade (T). Dessa forma, estabeleceu-se a pergunta norteadora: Quais os principais achados nos exames de neuroimagem e os desfechos no neurodesenvolvimento de crianças com Encefalopatia Hipóxico-Isquêmica?

Como critérios de elegibilidade, foram considerados: artigos de estudos de coorte e ensaios clínicos, publicados nos últimos cinco anos, idiomas em português, inglês e espanhol, lactente: nascimento-23 meses, lactente: 1-23 meses, humanos, diagnóstico por ressonância magnética, tomografia e eletroencefalograma. Foram excluídos os estudos com neonatos, animais e métodos diagnósticos que não incluíam exames de imagem, bem como, estudos secundários, trabalhos que não realizaram acompanhamento do neurodesenvolvimento, que não utilizaram escalas para avaliação do desenvolvimento infantil, que não apresentaram resultados dos achados neurológicos por exame de imagem e que analisaram somente a paralisia cerebral como desfecho.

A coleta de dados foi realizada nas bases de dados Cumulative Index to Nursing and Allied Health Literature (CINAHL) e National Library of Medicine, EUA (PubMed), em janeiro de 2021, através dos seguintes descritores próprios da CINAHL e dos termos Medical Subject Headings (MeSH): “asphyxia neonatorum”, "hypoxia-ischemia, brain”, "development”, “child development" e "image" articulados aos operadores booleanos “AND" e "OR". Na base CINAHL, utilizou-se como estratégia de busca: asphyxia neonatorum OR hypoxia-ischemia, brain AND development, totalizando 358 resultados; na PubMed, empregouse: asphyxia neonatorum OR hypoxia-ischemia, brain AND child development AND image, com um total de 1.914 resultados. Todos os resultados foram exportados para o Rayyan (Ouzzani, Hammady, Fedorowicz, \& Elmagarmid, 2016) aplicativo/website online e gratuito desenvolvido pelo Qatar Computing Research Institute (QCRI), para organização, armazenamento e seleção dos artigos.

Após a identificação dos estudos, a categorização foi realizada através de instrumento de elaboração própria, utilizando o software Excel, versão 2016, e estruturado em 11 variáveis: título, autor/ano, país, objetivos, tipo de estudo/nível de evidência, método, população estudada, tipo de exame de imagem, classificação da EHI, achados neurológicos nos exames de imagem e 
desfechos neurológicos.

\section{Resultados}

Para realizar a identificação dos principais desfechos neurológicos de crianças com EHI por meio dos achados na neuroimagem, a seleção dos estudos foi executada por meio da triagem dos títulos, leitura dos resumos e, por fim, análise na íntegra da publicação. A busca inicial identificou um total de 2.272 resultados nas bases de dados e um resultado por busca manual. Desses, foram excluídos 25 artigos por duplicidade através do Rayyan (QCRI) (Ouzzani, Hammady, Fedorowicz, \& Elmagarmid, 2016). Os títulos e resumos foram analisados por dois revisores independentes, que selecionaram 45 artigos para leitura na íntegra. Não foi necessária a participação do terceiro revisor. Após a leitura na íntegra, 11 artigos foram selecionados para a categorização. A estratégia de seleção está representada no fluxograma (Figura 1) segundo a recomendação PRISMA (Moher, Liberati, Tetzlaff, \& Altman, 2009). Os títulos e resumos foram analisados por dois revisores independentes, que selecionaram 45 artigos para leitura na íntegra. Não foi necessária a participação do terceiro revisor. Após a leitura na íntegra, 11 artigos foram selecionados para a categorização. 
Figura 1: Fluxograma com as etapas da Seleção de Artigos. Aracaju (SE), 2021.

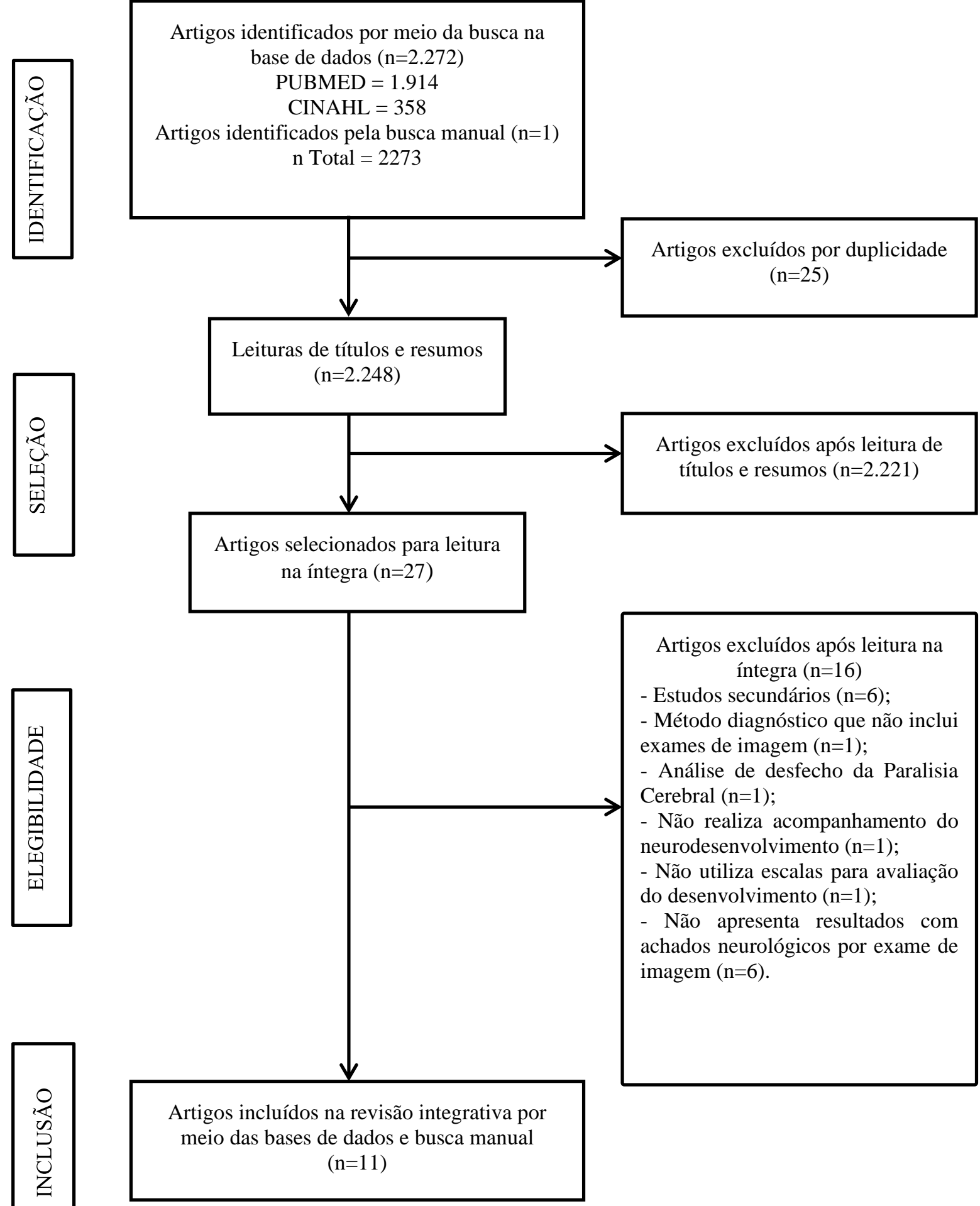

Fonte: Dados da pesquisa (2021).

Todos os estudos categorizados (Quadro 1), encontravam-se no idioma inglês, sendo três publicados na Inglaterra (Dunne et al., 2017; Mitra et al., 2018; Montaldo et al., 2018), dois no Brasil (Procianoy, Corso, Longo, Vedolin, \& Silveira, 2019; Procianoy et al., 2020), dois na Hungria (Barta et al., 2018; Lakatos et al., 2019), dois nos Estados Unidos (Goenka \& Yozawitz, 2018; Rao et al., 2019), um no Canadá (Al Amrani et al., 2017) e um na Itália (Mastrangelo et al., 2019). 
Quadro 1: Apresentação dos Estudos Selecionados para Categorização. Aracaju (SE), 2021.

\begin{tabular}{|c|c|c|c|}
\hline $\begin{array}{l}\text { AUTORES E ANO } \\
\text { DE PUBLICAÇÃO }\end{array}$ & TÍTULO DO ARTIGO & TIPO / NE & OBJETIVOS \\
\hline Al Amrani et al., 2017 & $\begin{array}{l}\text { Early Imaging and Adverse } \\
\text { Neurodevelopmental Out- } \\
\text { come in Asphyxiated New- } \\
\text { borns Treated With Hypo- } \\
\text { thermia }\end{array}$ & $\begin{array}{l}\text { Coorte Prospectiva } \\
\qquad \mathrm{NE}=3\end{array}$ & $\begin{array}{l}\text { Determinar se as alterações de difusão pre- } \\
\text { coce anormais em recém-nascidos tratados } \\
\text { com hipotermia estão associadas a resulta- } \\
\text { dos adversos do desenvolvimento neuroló- } \\
\text { gico aos } 2 \text { anos de idade. }\end{array}$ \\
\hline Barta et al., 2018. & $\begin{array}{l}\text { Prognostic value of early, } \\
\text { conventional proton mag- } \\
\text { netic resonance spectros- } \\
\text { copy in cooled asphyxiated } \\
\text { infants }\end{array}$ & $\begin{array}{c}\text { Análise Descritiva Re- } \\
\text { trospectiva } \\
\mathrm{NE}=3\end{array}$ & $\begin{array}{l}\text { Determinar o valor prognóstico de uma se- } \\
\text { quência EPRM completamente convencio- } \\
\text { nal (sem equipamento especial e técnicas } \\
\text { de pós-processamento além da análise bá- } \\
\text { sica fornecida pelo fornecedor), realizada } \\
\text { antes da } 96^{a} \text { hora de vida em bebês com } \\
\text { EHI, analisar várias proporções de metabó- } \\
\text { litos, sua dependência da idade e associa- } \\
\text { ção com o resultado do neurodesenvolvi- } \\
\text { mento de longo prazo. }\end{array}$ \\
\hline Dunne et al., 2017 & $\begin{array}{l}\text { Automated electroencepha- } \\
\text { lographic discontinuity in } \\
\text { cooled newborns predicts } \\
\text { cerebral MRI and neurode- } \\
\text { velopmental outcome }\end{array}$ & $\begin{array}{l}\text { Coorte } \\
\mathrm{NE}=3\end{array}$ & $\begin{array}{l}\text { O objetivo foi testar a hipótese de que uma } \\
\text { medida objetiva de descontinuidade do } \\
\text { EEG está associada com lesão do tecido ce- } \\
\text { rebral na ressonância magnética e resultado } \\
\text { do neurodesenvolvimento. }\end{array}$ \\
\hline $\begin{array}{c}\text { Goenka e Yozawitz, } \\
2018\end{array}$ & $\begin{array}{l}\text { Prognostic Markers in } \\
\text { Term Infants with Hy- } \\
\text { poxic-Ischemic Encepha- } \\
\text { lopathy: Comparative } \\
\text { Analysis of MRI, EEG, and } \\
\text { Apgar Scores }\end{array}$ & $\begin{array}{l}\text { Coorte } \\
\mathrm{NE}=3\end{array}$ & $\begin{array}{l}\text { Avaliar as sensibilidades e especificidades } \\
\text { de medidas individuais e combinadas para } \\
\text { determinar sua utilidade relativa como pre- } \\
\text { ditores de resultados neurológicos de longo } \\
\text { prazo para bebês com EHI. }\end{array}$ \\
\hline Lakatos et al., 2019 & $\begin{array}{l}\text { Neurodevelopmental effect } \\
\text { of intracranial hemorrhage } \\
\text { observed in hypoxic is- } \\
\text { chemic brain injury in hy- } \\
\text { pothermia-treated asphyxi- } \\
\text { ated neonates - an MRI } \\
\text { study }\end{array}$ & $\begin{array}{l}\text { Coorte Observacional } \\
\text { Retrospectiva } \\
\mathrm{NE}=3\end{array}$ & $\begin{array}{l}\text { Determinar se a presença de EHI na RM } \\
\text { precoce juntamente com os sinais de EHI } \\
\text { têm um impacto no prognóstico em neona- } \\
\text { tos resfriados com o diagnóstico clínico de } \\
\text { EHI. }\end{array}$ \\
\hline $\begin{array}{l}\text { Mastrangelo et al., } \\
2019\end{array}$ & $\begin{array}{l}\text { Early Post-cooling Brain } \\
\text { Magnetic Resonance for } \\
\text { the Prediction of Neurode- } \\
\text { velopmental Outcome in } \\
\text { Newborns with Hypoxic-- } \\
\text { Ischemic Encephalopathy }\end{array}$ & $\begin{array}{l}\text { Coorte Retrospectiva } \\
\qquad \mathrm{NE}=3\end{array}$ & $\begin{array}{l}\text { Avaliar o papel preditivo dos principais ín- } \\
\text { dices estruturais, de difusividade e espec- } \\
\text { troscópicos na ressonância magnética cere- } \\
\text { bral pós-resfriamento precoce para o resul- } \\
\text { tado do desenvolvimento em recém-nasci- } \\
\text { dos com EHI. }\end{array}$ \\
\hline Mitra et al., 2018 & $\begin{array}{l}\text { Proton magnetic resonance } \\
\text { spectroscopy lactate/N- } \\
\text { acetylaspartate within } 2 \\
\text { weeks of birth accurately } \\
\text { predicts 2-year motor, cog- } \\
\text { nitive and language out- } \\
\text { comes in neonatal encepha- } \\
\text { lopathy after therapeutic } \\
\text { hypothermia }\end{array}$ & $\begin{array}{l}\text { Estudo Retrospectivo } \\
\qquad \mathrm{NE}=3\end{array}$ & $\begin{array}{l}\text { Avaliar, em bebês com encefalopatia neo- } \\
\text { natal que foram resfriados: (1) as razões de } \\
\text { Lac+Thr/ tNAA na EPRM em GBT sensi- } \\
\text { bilidade e especificidade em 3T para resul- } \\
\text { tados motores, cognitivos e de linguagem } \\
\text { Bayley III em } 2 \text { anos; (2) previsão do resul- } \\
\text { tado motor de Lac + Thr/tNAA versus ou- } \\
\text { tros biomarcadores de ressonância magné- } \\
\text { tica antes da pseudonormalização; (3) Cor- } \\
\text { relação BGT Lac + Thr/tNAA com a pon- } \\
\text { tuação de IRM do NICHD. }\end{array}$ \\
\hline
\end{tabular}




\begin{tabular}{|c|c|c|c|}
\hline Montaldo et al., 2018 & $\begin{array}{l}\text { Therapeutic hypothermia } \\
\text { initiated within } 6 \text { hours of } \\
\text { birth is associated with re- } \\
\text { duced brain injury on MR } \\
\text { biomarkers in mild hy- } \\
\text { poxic-ischaemic encepha- } \\
\text { lopathy: a non-randomised } \\
\text { cohort study }\end{array}$ & $\begin{array}{l}\text { Estudo Observacional } \\
\qquad \mathrm{NE}=3\end{array}$ & $\begin{array}{l}\text { Examinar o efeito da hipotermia de corpo } \\
\text { inteiro sobre biomarcadores de RM cere- } \\
\text { bral e resultados neurológicos em } 2 \text { anos } \\
\text { após EHI leve. }\end{array}$ \\
\hline Procianoy et al., 2019 & $\begin{array}{l}\text { Therapeutic hypothermia } \\
\text { for neonatal hypoxic-is- } \\
\text { chemic encephalopathy: } \\
\text { magnetic resonance imag- } \\
\text { ing findings and neurologi- } \\
\text { cal outcomes in a Brazilian } \\
\text { cohort }\end{array}$ & $\begin{array}{l}\text { Coorte } \\
\mathrm{NE}=3\end{array}$ & $\begin{array}{l}\text { Determinar o resultado do neurodesenvol- } \\
\text { vimento neonatal com EHI moderado a } \\
\text { grave devido à AP após hipotermia de } \\
\text { corpo inteiro e identificar os achados de } \\
\text { ressonância magnética do cérebro associa- } \\
\text { dos aos resultados neurológicos e com o } \\
\text { grau de EHI em conjunto brasileiro. }\end{array}$ \\
\hline Procianoy et al., 2020 & $\begin{array}{l}\text { Outcome and Feasibility af- } \\
\text { ter } 7 \text { Years of Therapeutic } \\
\text { Hypothermia in Southern } \\
\text { Brazil }\end{array}$ & $\begin{array}{l}\text { Coorte } \\
\mathrm{NE}=3\end{array}$ & $\begin{array}{l}\text { Descrever a experiência em uma unidade } \\
\text { de terapia intensiva neonatal nível III de um } \\
\text { país em desenvolvimento, utilizando proto- } \\
\text { colo bem estabelecido, relatando as carac- } \\
\text { terísticas dos pacientes tratados, efeitos ad- } \\
\text { versos, resultados e neurodesenvolvimento } \\
\text { a longo prazo. }\end{array}$ \\
\hline Rao et al., 2019 & $\begin{array}{l}\text { Neurodevelopmental Out- } \\
\text { comes in Neonates with } \\
\text { Mild Hypoxic Ischemic En- } \\
\text { cephalopathy Treated with } \\
\text { Therapeutic Hypothermia }\end{array}$ & $\begin{array}{l}\text { Coorte Retrospectiva } \\
\qquad \mathrm{NE}=3\end{array}$ & $\begin{array}{l}\text { Investigar o curso clínico e os resultados do } \\
\text { neurodesenvolvimento de uma coorte de } \\
\text { lactentes com EHI leve que foram submeti- } \\
\text { dos à HT, comparando com os resultados } \\
\text { do neurodesenvolvimento de uma coorte } \\
\text { local com lactentes sem EHI. }\end{array}$ \\
\hline
\end{tabular}

Legenda: NE = Nível de Evidência; EPRM = Espectroscopia de Prótons por Ressonância Magnética; EHI = Encefalopatia Hipóxico-Isquêmica; EEG = Eletroencefalograma; RM = Ressonância Magnética; Lac = Lactato; Thr = Metil da Treonina; tNAA = N-Acetil-Aspartato total; BGT = Gânglios da Base e Tálamo; IRM = Imagem por Ressonância Magnética; NICHD = National Institute Of Child Health And Human Development; AP = Asfixia Perinatal; HT = Hipotermia Terapêutica; Fonte: Dados da pesquisa (2021).

A maior parte dos estudos incluíram crianças com os graus moderado e grave da EHI, ao passo que dois fizeram a análise apenas de crianças com encefalopatia leve (Montaldo et al., 2018; Rao et al., 2019). Com relação aos critérios para avaliação do grau da EHI, estes variaram conforme os autores, de forma que sete estudos utilizaram a classificação de Sarnat e Sarnat (Barta et al., 2018; Goenka \& Yozawitz, 2018; Mastrangelo et al., 2019; Mitra et al., 2018; Montaldo et al., 2018; Procianoy et al., 2019; Procianoy et al., 2020). Um dos estudos evidenciou o grau da EHI através de um exame neurológico padrão institucional, somado ao EEG de amplitude integrada (Al Amrani et al., 2017), enquanto outro avaliou os bebês quanto ao estado de hiperalerta com resposta exagerada a excitação, além do EEG (Rao et al., 2019).

Além disso, dois artigos utilizaram os critérios do estudo TOBY (Total Body Hypothermia for Neonatal Encephalopathy Trial), que classificou a EHI em grave e moderada, indicados pelo nível de consciência (letargia, estupor ou coma), hipotonia, reflexos anormais (incluindo anormalidades oculomotoras ou pupilares), sucção ausente/fraca ou convulsões clínicas, além da avaliação do EEG (Dunne et al., 2017; Lakatos et al., 2019).

Todos os artigos categorizados utilizaram a ressonância magnética como exame para avaliação das estruturas e, além dessa técnica, cinco estudos realizaram a avaliação das lesões por meio da espectroscopia de ressonância magnética (Barta et al., 2018; Lakatos et al., 2019; Mastrangelo et al., 2019; Mitra et al., 2018; Montaldo et al., 2018). A grande maioria dos estudos afirma que a ressonância magnética, bem como suas técnicas, são bons preditores de desfecho neurológico adverso. Apenas três estudos não observaram esse aspecto por não ser objetivo dos mesmos (Montaldo et al., 2018; Procianoy et al., 2020; Rao et al., 2019). 
Quanto às alterações encontradas por meio dos exames de neuroimagem, os estudos avaliaram as estruturas encefálicas e os resultados identificaram diferentes achados e desfechos neurológicos, conforme o local do acometimento (Quadro 2).

Quadro 2: Descrição dos Achados na Ressonância Magnética e Desfechos Neurológicos. Aracaju (SE), 2021.

\begin{tabular}{|c|c|c|}
\hline $\begin{array}{l}\text { AUTORES E } \\
\text { ANO DE PUBLI- } \\
\text { CAÇÃO }\end{array}$ & $\begin{array}{l}\text { ACHADOS NEUROLÓGI- } \\
\text { COS }\end{array}$ & DESFECHOS NEUROLÓGICOS \\
\hline $\begin{array}{l}\text { Al Amrani et al., } \\
\qquad 2017\end{array}$ & $\begin{array}{l}\text { Injúrias em GB e em bacia hi- } \\
\text { drográfica. }\end{array}$ & $\begin{array}{l}\text { Das } 29 \text { crianças avaliadas, cinco desenvolveram paralisia cerebral, } \\
\text { atraso de desenvolvimento global e/ou epilepsia. De acordo com } \\
\text { GMFCS, os níveis de paralisia das crianças variaram: nível } 1 \text { (1/5), ní- } \\
\text { vel } 2(2 / 5) \text {, nível } 4(1 / 5) \text { e nível } 5(1 / 5) \text {. Em relação aos atrasos foram } \\
\text { classificados: atraso de desenvolvimento global incluiu atraso motor } \\
\text { grosso }(4 / 5) \text {, atraso motor fino }(5 / 5) \text {, atraso de linguagem expressiva } \\
(5 / 5) \text {, atraso de linguagem receptiva (3/5). Além de traços de autismo } \\
(2 / 5) \text { e traços de transtorno de déficit de atenção/hiperatividade }(1 / 5) \text {. }\end{array}$ \\
\hline Barta et al., 2018. & $\begin{array}{l}\text { Insultos em GB e PLIC apon- } \\
\text { tados como mais comuns, com } \\
\text { alterações subsequentes em } \\
\text { tálamo, SB e córtex. }\end{array}$ & $\begin{array}{l}\text { De } 51 \text { crianças avaliadas, } 16 \text { apresentaram resultados ruins, nove cri- } \\
\text { anças foram a óbito no período perinatal e sete tiveram desenvolvi- } \\
\text { mento moderado/gravemente atrasado. Destas, quatro apresentaram } \\
\text { paralisia cerebral, duas retardo mental e um sofreu perda auditiva neu- } \\
\text { ronal e epilepsia. }\end{array}$ \\
\hline Dunne et al., 2017 & GBT, PLIC e SB. & $\begin{array}{l}\text { Das } 43 \text { crianças avaliadas no acompanhamento, } 14 \text { crianças apresenta- } \\
\text { ram desfechos desfavoráveis: quatro morreram, seis tiveram paralisia } \\
\text { cerebral, duas necessitaram de aparelhos auditivos bilaterais para perda } \\
\text { auditiva neurossensorial profunda, uma teve autismo, convulsões e } \\
\text { atraso global da fala e uma teve convulsões e perda de audição unila- } \\
\text { teral. }\end{array}$ \\
\hline $\begin{array}{l}\text { Goenka e Yo- } \\
\text { zawitz, } 2018\end{array}$ & GB ou tálamo. & $\begin{array}{l}\text { Das } 46 \text { crianças avaliadas, } 23 \text { apresentaram algum déficit neurológico } \\
\text { ou morte, sem especificar os domínios alterados. }\end{array}$ \\
\hline $\begin{array}{l}\text { Lakatos et al., } \\
\qquad 2019\end{array}$ & $\begin{array}{l}\text { GBT, bacia hidrográfica e leu- } \\
\text { comalácia periventricular he- } \\
\text { morrágica. }\end{array}$ & $\begin{array}{l}\text { Dos } 99 \text { pacientes analisados, } 27 \text { tiveram desenvolvimento levemente } \\
\text { atrasado e } 12 \text { apresentaram atraso significativo, sendo sete desses di- } \\
\text { agnosticados com paralisia cerebral. Analisando separadamente, no } \\
\text { Grupo com EHI e Hemorragia Intracraniana, } 12 \text { crianças apresentaram } \\
\text { um resultado adverso e no Grupo que apresentava somente EHI, } 33 \\
\text { pacientes demonstraram resultado adverso. }\end{array}$ \\
\hline $\begin{array}{l}\text { Mastrangelo et al., } \\
2019\end{array}$ & GB e área periventricular. & $\begin{array}{l}\text { O estudo analisou que aos } 12 \text { meses o grupo estudado apresentou re- } \\
\text { sultado ruim, com piores desfechos em coordenação de mãos e olhos, } \\
\text { audição e fala, continuando com avaliação desfavorável aos } 24 \text { meses } \\
\text { de idade. }\end{array}$ \\
\hline Mitra et al., 2018 & $\begin{array}{l}\text { Devastação hemisférica mais } \\
\text { prevalente, seguida de GBT, } \\
\text { ALIC, PLIC e lesões cerebrais } \\
\text { extensas e mínimas. }\end{array}$ & $\begin{array}{l}\text { De um total de } 24 \text { crianças analisadas, } 18 \text { apresentaram resultados } \\
\text { anormais nos três domínios (cognitivo, motor e de linguagem). As de- } \\
\text { mais, apresentaram anormalidade apenas em um domínio, uma criança } \\
\text { no cognitivo, duas no motor e três no de linguagem. }\end{array}$ \\
\hline $\begin{array}{l}\text { Montaldo et al., } \\
\quad 2018\end{array}$ & $\begin{array}{l}\text { Sinal de anormalidade leve e } \\
\text { moderada na substância } \\
\text { branca e alterações de baixa } \\
\text { relevância em córtex e GBT. }\end{array}$ & $\begin{array}{l}\text { Dos } 40 \text { bebês acompanhados, apenas duas crianças, ambas do Grupo } \\
\text { sem HT ou com HT } \leq 12 \mathrm{~h} \text {, apresentaram deficiência no desenvolvi- } \\
\text { mento. Em uma observou-se deficiência motora leve sem paralisia ce- } \\
\text { rebral e grave atraso de linguagem, e a outra teve deficiência leve nos } \\
\text { domínios cognitivo, motor e de linguagem e monoplegia de braço di- } \\
\text { reito. }\end{array}$ \\
\hline $\begin{array}{l}\text { Procianoy et al., } \\
\quad 2019\end{array}$ & $\begin{array}{l}\text { GBT, SB, PLIC e lesão corti- } \\
\text { cal. }\end{array}$ & $\begin{array}{l}\text { Houve associação entre o desenvolvimento motor anormal e a EHI } \\
\text { grave pelas escalas AIMS e GMFCS. Já com a escala de Bayley III, o } \\
\text { estudo apontou que o atraso do desenvolvimento motor está associado } \\
\text { à EHI grave e a presença de lesão em PLIC. O menor escore da escala } \\
\text { cognitiva também foi associado à lesão em PLIC e da escala de lingua- } \\
\text { gem, as menores pontuações, estavam associadas à presença de lesão } \\
\text { em PLIC, GBT e lesão cortical. }\end{array}$ \\
\hline
\end{tabular}




\begin{tabular}{|c|l|l|}
\hline $\begin{array}{c}\text { Procianoy } \text { et al., } \\
2020\end{array}$ & $\begin{array}{l}\text { GBT, seguida de PLIC, SB e } \\
\text { lesão cortical. }\end{array}$ & $\begin{array}{l}\text { Dos } 40 \text { pacientes avaliados aos 12 meses, 18 tiveram atrasos, desses, } \\
13 \text { no domínio cognitivo, oito no domínio motor, 15 no de linguagem. }\end{array}$ \\
\hline Rao et al., 2019 & $\begin{array}{l}\text { Bacia hidrográfica, SB, subs- } \\
\text { tância cinzenta nuclear e lesão } \\
\text { global, respectivamente. }\end{array}$ & $\begin{array}{l}\text { Nesse estudo, foi comparada as médias dos resultados nos três domí- } \\
\text { nios, pela Escala de Bayley III, entre dois grupos (Grupo HT e Con- } \\
\text { trole) e observou-se que a diferença dos resultados foi insignificante e } \\
\text { que ambos tiveram uma boa avaliação. }\end{array}$ \\
\hline
\end{tabular}

Legenda: GB = Gânglios da Base; GMFCS = Sistema de Classificação da Função Motora Grossa; PLIC = Membro Posterior da Cápsula Interna; GBT = Gânglios da Base e Tálamo; SB = Substância Branca; EHI = Encefalopatia Hipóxico-Isquêmica; ALIC = Membro Anterior da Cápsula Interna; HT = Hipotermia Terapêutica; AIMS = Alberta Infant Motor Scale; Fonte: Dados da pesquisa (2021).

Dentre os principais desfechos neurológicos encontrados, destacam-se o atraso motor, cognitivo e de linguagem. Bem como, a gravidade das sequelas neurológicas com a complicação da paralisia cerebral e da ocorrência do óbito em algumas crianças. O quadro 2 reúne as informações dos estudos no que diz respeito aos desfechos neurológicos das crianças com AP.

Dos estudos categorizados, oito descreveram correlação entre a mortalidade e o grau de encefalopatia, de forma que os óbitos das crianças ocorreram naquelas com EHI moderada a grave (Al Amrani et al., 2017; Barta et al., 2018; Dunne et al., 2017; Goenka \& Yozawitz, 2018; Lakatos et al., 2019; Mitra et al., 2018; Procianoy et al., 2019; Procianoy et al., 2020). Quanto aos demais estudos, não há resultados sobre a mortalidade das crianças, inclusive entre os que trabalharam apenas com EHI leve (Mastrangelo et al., 2019; Montaldo et al., 2018; Rao et al., 2019).

Todos os estudos realizaram o acompanhamento das crianças para avaliação do desenvolvimento infantil, sendo utilizada na maioria dos artigos a Escala Bayley de Desenvolvimento do Bebê e da Criança Pequena, terceira edição - Bayley-III, que avalia o desenvolvimento infantil e identifica possíveis problemas dentro de cinco domínios: cognitivo, linguístico, motor, socioemocional e comportamento adaptativo (Bayley, 2006). Além dessa escala, alguns estudos também utilizaram concomitantemente ou isoladamente as Escalas de Desenvolvimento Mental Griffiths - $3^{\text {a }}$ edição (GMDS-III) (Green et al., 2016), Alberta Infant Motor Scale (AIMS) (Piper \& Darrah, 1994), Sistema de Classificação da Função Motora Grossa (GMFCS) (Palisano et al., 1977), Avaliação pelo Center for Disease Control, Royal College of Paediatrics and Child Health (American Academy of Pediatrics, 2007), que também são instrumentos para avaliação do desenvolvimento da criança.

\section{Discussão}

Esta pesquisa objetivou analisar os principais achados nos exames de neuroimagem e os desfechos no neurodesenvolvimento em crianças com EHI. Os estudos utilizaram a ressonância magnética como principal exame de imagem para identificação de lesões, sendo um bom preditor do neurodesenvolvimento das crianças. Esta técnica, além de não ser invasiva e não utilizar radiação ionizante, produz imagens fidedignas em três dimensões e possui ótima resolução espacial e de contraste (Montanha, Silva Filho, \& Frazão, 2020; Guimarães et al., 2014). Ademais, diversas técnicas podem ser empregadas com diferentes finalidades de visualização, como a difusão, a perfusão e a espectroscopia, que auxiliam na detecção de outros tipos de anormalidades, como os níveis bioquímicos no tecido cerebral (Guimarães et al., 2014).

A ressonância magnética realizada na primeira semana de vida e após o sétimo dia de vida em neonato com EHI demonstrou ser bom preditor de morte ou incapacidade grave aos 24 meses de idade. Ressalta-se, ainda, que ao ser realizada precocemente, apresentou mais especificidade em comparação ao exame após o sétimo dia. Todavia, esta se torna fundamental para confirmar lesões sutis que não foram detectadas anteriormente na primeira semana (Charon et al., 2016). Apesar da importância dos exames de imagem, os países em desenvolvimento possuem acesso limitado a esses recursos, e dessa forma, a avaliação e diagnóstico de cunho neurológico tornam-se muitas vezes impossibilitados, trazendo déficit à assistência e pouca informação na literatura pediátrica (Azhari et al., 2020). 
Nas imagens da ressonância magnética, as lesões foram identificadas em diferentes estruturas encefálicas, sendo as mais frequentes: gânglios da base e tálamo, substância branca, córtex cerebral, membro posterior da cápsula interna e bacia hidrográfica. As estruturas mais acometidas nas crianças com AP foram os gânglios da base e tálamo e substância branca

Os gânglios da base referem-se a um conjunto de núcleos que desempenham inúmeras e complexas funções no corpo humano, dentre elas, o papel essencial no controle do movimento voluntário. Portanto, afecções associadas a esses núcleos estão relacionadas a acometimentos motores. Existe também um alto grau de comunicação entre os gânglios da base e outras estruturas, como tálamo e córtex cerebral, para o desempenho dessa e de outras atividades, como as funções cognitiva, sensitivas e comportamentais (Alves, 2012).

As lesões em substância branca demonstraram associação com o déficit cognitivo (Zhong et al., 2017). Ressalta-se ainda que lesões moderadas e graves nos gânglios da base e tálamo, assim como lesões graves na substância branca estão associadas à paralisia cerebral (Tagin, Woolcott, Vincer, Whyte, \& Stinson, 2012). Dessa forma, diante da quantidade de funções desempenhadas por essas estruturas, que são as mais acometidas na AP, evidencia-se uma grande variedade de manifestações clínicas nessas crianças, com destaque aos atrasos motor e cognitivo.

Em seu estudo, Ferreira (2019) corrobora esses achados ao verificar que a maioria das crianças com EHI apresentou alguma alteração no neurodesenvolvimento, destas a epilepsia e paralisia cerebral foram as duas comorbidades mais prevalentes, seguidas de alterações cognitivas que foram analisadas na avaliação do desenvolvimento e do comportamento adaptativo. Outras pesquisas também apontaram a associação da EHI com alterações motoras (Adhikari \& Rao, 2017; Araújo, Eickmann, \& Coutinho, 2013).

Em relação à análise desses desfechos neurológicos com o grau da EHI, é sabido que há uma forte correlação entre ambos. Assim, quanto maior o grau da lesão, maior é a incidência de morte ou deficiência grave (Edwards et al., 2010). A forma leve da EHI geralmente resulta em recuperação total das lesões ou distúrbios psicomotores leves, enquanto as formas moderadas e graves trazem consequências mais severas, por estarem associadas a uma maior quantidade de morte neuronal em córtex e substância cinzenta (Iwata et al., 2007; Papazian, 2018).

Sobre a avaliação desses comprometimentos neurológicos, destaca-se a variedade na utilização de critérios para identificar o desenvolvimento das crianças após a EHI. Essa falta de padronização pode implicar em diferentes resultados nas pesquisas, além de dificultar o planejamento na prática clínica (Albuquerque \& Cunha, 2020; Takazono \& Golin, 2013), uma vez que a identificação precoce de alterações é de grande relevância para o levantamento de dados clínicos e para uma adequada intervenção. Além disso, o reconhecimento correto do quadro neurológico também contribui para a diminuição do tempo hospitalar, redução dos gastos e garantia de um acompanhamento especializado pós-alta (Freitas, Pereira \& Oliveira, 2018).

Existe na literatura pediátrica uma gama de instrumentos que auxiliam na mensuração e avaliação de possíveis atrasos infantis (Silva et al., 2011). A padronização da avaliação do desenvolvimento infantil é possibilitada graças ao uso dessas escalas que oportunizam uma análise sistematizada, comparam o desenvolvimento de determinado grupo à norma padrão e subsidiam os profissionais da saúde em suas condutas (Cardoso, Formiga, Bizinotto, Tessler, \& Rosa Neto, 2017). É válido ressaltar, que tais instrumentos são desenvolvidos em alguns países, o que os torna inadequados para populações diferentes, sendo necessário o processo de adaptação e validação para o uso em outros locais (Albuquerque \& Cunha, 2020; Madaschi, 2012; Rodrigues, 2012)

O acompanhamento do desenvolvimento infantil foi realizado em todos os estudos categorizados nessa revisão até os dois anos de idade. A respeito disso, destaca-se que o seguimento do cuidado em crianças de alto risco, o "follow-up", é de grande importância para acompanhar o neurodesenvolvimento e promover intervenções precoces após a alta hospitalar (Ballantyne, Stevens, Guttmann, Willan, \& Rosenbaum, 2014; Souza et al., 2020). Vale ressaltar que os profissionais da Atenção Básica de Saúde possuem participação fundamental na vigilância dos atrasos neuropsicomotores, durante as consultas de puericultura, 
de maneira que trabalham na identificação dessas crianças e no encaminhamento aos serviços especializados (Araújo, Gerzson, \& Aldeida, 2020). Esses serviços devem contar com uma equipe multidisciplinar (Freitas, 2016) com papeis bem estabelecidos para atuar na orientação e estimulação precoce, com o objetivo de minimizar complicações que podem afetar negativamente a qualidade de vida dessas crianças e de toda sua família (Souza et al., 2020).

\section{Considerações Finais}

A pesquisa evidenciou a existência de lesões em diferentes estruturas encefálicas por meio da ressonância magnética e salientou uma maior frequência de insultos nos Gânglios da Base e Tálamo e Substância Branca. Bem como, apresentou a neuroimagem como um método para avaliação das estruturas nervosas e detecção das anormalidades. Em relação ao acompanhamento do desenvolvimento infantil, os principais desfechos neurológicos identificados foram atraso motor, cognitivo e de linguagem. Ademais, a maior parte dos estudos afirma que a neuroimagem por ressonância magnética, e suas diversas técnicas, são bons preditores do neurodesenvolvimento de crianças acometidas por EHI.

As limitações verificadas foram ausência ou baixo acesso aos exames neurológicos de imagem em países subdesenvolvidos, dificultando a avaliação precisa dessas crianças. Além disso, embora a ressonância magnética demonstre uma boa predição para resultados adversos, destaca-se que não é possível associar a lesão neurológica ao tipo de neurodeficiência.

Ressalta-se, ainda, que as escalas de avaliação do desenvolvimento infantil são elaboradas em países que possuem um perfil populacional distinto às particularidades sociodemográficas de países em desenvolvimento, o que pode acarretar equívocos na avaliação dos resultados infantis. Portanto, torna-se necessária a adaptação e validação de instrumentos padronizados que supram as necessidades e especificidades de cada local. Ademais, esses escores clínicos favorecem a identificação precoce dos atrasos neurológicos, que podem ser aplicados na Atenção Primária e possuem fácil acesso e baixo custo.

Nesse tocante, sugere-se a elaboração de estudos futuros acerca da padronização dos instrumentos de avaliação do desenvolvimento infantil de acordo com o perfil populacional brasileiro, bem como a viabilidade da utilização dos mesmos nos cuidados de saúde primários.

\section{Referências}

Abreu, A. J. P. (2018). Encefalopatia Neonatal: etiologia e morbilidade [Dissertação de Mestrado, Faculdade de Medicina da Universidade de Coimbra]. Repositório científico da Universidade de Coimbra. http://hdl.handle.net/10316/82130

Adhikari, S., \& Rao, K. S. (2017). Neurodevelopmental outcome of term infants with perinatal asphyxia with hypoxic ischemic encephalopathy stage II. Brain \& development, 39(2), 107-111. https://doi.org/10.1016/j.braindev.2016.09.005

Al Amrani, F., Kwan, S., Gilbert, G., Saint-Martin, C., Shevell, M., \& Wintermark P. (2017). Early Imaging and Adverse Neurodevelopmental Outcome in Asphyxiated Newborns Treated With Hypothermia. Pediatric neurology, 73, 20-27. https://doi.org/10.1016/j.pediatrneurol.2017.04.025

Albuquerque, K. A., \& Cunha, A. C. B. da. (2020). Novas tendências em instrumentos para triagem do desenvolvimento infantil no Brasil: uma revisão sistemática. Journal of Human Growth and Development, 30(2), 188-196. https://doi.org/10.7322/jhgd.v30.10366

Alves, S. C. C. (2012). Fisiopatologia dos gânglios da base na Doença de Parkinson [Dissertação de Mestrado, Faculdade de Medicina da Universidade de Coimbra]. Repositório científico da Universidade de Coimbra. http://hdl.handle.net/10316/84946

American Academy of Pediatrics. (2007). Bright futures: Guidelines for health supervision of infants, children, and adolescents (3a ed.). American Academy of Pediatrics.

Araújo, A. T. C., Eickmann, S. H., \& Coutinho, S. B. (2013). Fatores associados ao atraso do desenvolvimento motor de crianças prematuras internadas em unidade de neonatologia. Revista Brasileira de Saúde Materno Infantil, 13(2), 119-128. https://doi.org/10.1590/S1519-38292013000200005

Araújo, B. C., Gerzson, L. R., \& Almeida, C. S. de. (2020). Aspectos avaliativos do desenvolvimento infantil na atenção básica: uma revisão integrativa. Archives of Health Sciences, 27(1), 56-60. https://doi.org/10.17696/2318-3691.27.1.2020.1462 
Azhari, A., Truzzi, A., Neoh, M. J.-Y., Balagtas, J. P. M., Tan, H. H., Goh, P. P., Ang, X. A., Setoh, P., Rigo, P., Bornstein, M. H., \& Esposito, G. (2020). A decade of infant neuroimaging research: What have we learned and where are we going? Infant Behavior and Development, 58, 1-15. https://doi.org/10.1016/j.infbeh.2019.101389

Ballantyne, M., Stevens, B., Guttmann, A., Willan, A. R., \& Rosenbaum, P. (2014). Maternal and infant predictors of attendance at Neonatal Follow-Up programmes. Child: care, health and development, $40(2), 250-258$. https://doi.org/10.1111/cch.12015

Ballot, D. E., Rakotsoane, D., Cooper, P. A., Ramdin, T. D., Chirwa, T., \& Pepper, M. S. (2020). A prospective observational study of developmental outcomes in survivors of neonatal hypoxic ischaemic encephalopathy in South Africa. The South African Medical Journal, 110(4), 308-312. https://doi.org/10.7196/SAMJ.2020.v110i4.14311

Barta, H., Jermendy, A., Kolossvary, M., Kozak, L. R., Lakatos, A., Meder, U., Szabo, M., \& Rudas, G. (2018). Prognostic value of early, conventional proton magnetic resonance spectroscopy in cooled asphyxiated infants. BMC Pediatrics, 18(302), 1-11. https://doi.org/10.1186/s12887-018-1269-6

Bayley, N. (2006). Bayley scales of Infant and Toddler Development (3a ed.). Harcourt Assessment.

Binkowski, R. T. K., \& Weinmann, A. R. M. (2015). Hipotermia terapêutica em recém-nascidos com diagnóstico de encefalopatia hipóxico isquêmica: Revisão de Literatura. Revista Saúde (Santa Maria), 41(1), 37-48. http://dx.doi.org/10.5902/2236583410649

Botelho, L. L. R., Cunha, C. C. A., \& Macedo, M. (2011). O método da revisão integrativa nos estudos organizacionais. Gestão E Sociedade, 5(11), 121-136. https://doi.org/10.21171/ges.v5i11.1220

Cardoso, F. G. C., Formiga, C. K. M. R., Bizinotto, T., Tessler, R. B., \& Rosa Neto, F. (2017). Validade concorrente da escala Brunet-Lézine com a escala bayley para avaliação do desenvolvimento de bebês pré-termo até dois anos. Revista Paulista de Pediatria, 35(2), 144-150. https://doi.org/10.1590/1984$0462 / ; 2017 ; 35 ; 2 ; 00005$

Charon, V., Proisy, M., Bretaudeau, G., Bruneau, B., Pladys, P., Beuchée, A., Burnouf-Rose, G., Ferré, J.-C., \& Rozel, C. (2016). Early MRI in neonatal hypoxicischaemic encephalopathy treated with hypothermia: Prognostic role at 2-year follow-up. European journal of radiology, 85(8), 1366-1374. https://doi.org/10.1016/j.ejrad.2016.05.005

Dunne, J. M., Wertheim, D., Clarke, P., Kapellou, O., Chisholm, P., Boardman, J. P., \& Shah, D. K. (2017). Automated electroencephalographic discontinuity in cooled newborns predicts cerebral MRI and neurodevelopmental outcome. Archives of Disease in Childhood - Fetal and Neonatal Edition, $102(1)$, 58-64. https://doi.org/10.1136/archdischild-2015-309697

Edwards, A. D., Brocklehurst, P., Gunn, A. J., Halliday, H., Juszczak, E., Levene, M., Strohm, B., Thoresen, M., Whitelaw, A., \& Azzopardi, D. (2010). Neurological outcomes at 18 months of age after moderate hypothermia for perinatal hypoxic ischaemic encephalopathy: synthesis and meta-analysis of trial data. $B M J, 340$ (c363), 1-7. https://doi.org/10.1136/bmj.c363

Ferreira, L. S. (2019). Alterações de neuroimagem estrutural em crianças com encefalopatia epiléptica de causa desconhecida. [Dissertação de Mestrado, Faculdade de Ciências Médicas da Universidade Estadual de Campinas]. Repositório da Produção Científica e Intelectual da Unicamp. http://repositorio.unicamp.br/jspui/handle/REPOSIP/334047

França, E. B., Lansky, S., Rego, M. A. S., Malta, D. C., França, J. S., Teixeira, R., Porto, D., Almeida, M. F., Souza, M. F. M., Szwarcwald, C. L., Mooney, M., Naghavi, M., \& Vasconcelos, A. M. N. (2017). Principais causas da mortalidade na infância no Brasil, em 1990 e 2015: estimativas do estudo de Carga Global de Doença. Revista Brasileira de Epidemiologia, 20(1), 46-60. https://doi.org/10.1590/1980-5497201700050005

Freitas, Z. M. P. (2016). Influência da avaliação neurológica seriada e seus reflexos no prognóstico funcional de recém-nascidos a termo com asfixia perinatal [Tese de Doutorado, Universidade Federal de Sergipe]. Biblioteca Digital de Teses e Dissertações (BDTD) da UFS. https://ri.ufs.br/jspui/handle/riufs/3634

Freitas, Z. M. P., Pereira, C. U., \& Oliveira, D. M. P. (2018). Influência da avaliação neurológica seriada durante período de internamento hospitalar e seus reflexos no prognóstico funcional de recém-nascidos a termo com asfixia perinatal. Revista Brasileira de Neurologia e Psiquiatria, 22(2), 142-156. https://www.revneuropsiq.com.br/rbnp/article/view/409

Goenka, A., \& Yozawitz, E. (2018). Prognostic Markers in Term Infants with Hypoxic-Ischemic Encephalopathy: Comparative Analysis of MRI, EEG, and Apgar Scores. Journal of Pediatric Neurology, 16(1), 8-14. https://doi.org/10.1055/s-0037-1606367

Green, E., Stroud, L., Bloomfield, S., Cronje, J., Foxcroft, C., Hurter, K., Lane, H., Marais, R., Marx, C., McAlinden, P., O'Connell, R., Paradice, R., \& Venter, D. (2018). Manual Griffiths III - Griffiths Scales of Child Development (3a ed.). Hogrefe Editora.

Guimarães, M. D., Schuch, A., Hochhegger, B., Gross, J. L., Chojniak, R., \& Marchiori, E. (2014). Ressonância magnética funcional na oncologia: estado da arte. Radiologia Brasileira, 47(2), 101-111. https://doi.org/10.1590/S0100-39842014000200013

Gunn, A. J., \& Thoresen, M. (2019). Neonatal encephalopathy and hypoxic-ischemic encephalopathy. In L. S. de Vries, \& H. C. Glass (Eds.) Handbook of Clinical Neurology (Vol. 162, Cap.10, pp. 217-237). Elsevier. https://doi.org/10.1016/B978-0-444-64029-1.00010-2

Iwata, O., Iwata, S., Thornton, J. S., Vita, E. de., Bainbridge, A., Herbert, L., Scaravilli, F., Peebles, D., Wyatt, J. S., Candy, E. B., \& Robertson, N. J. (2007). "Therapeutic time window" duration decreases with increasing severity of cerebral hypoxia-ischaemia under normothermia and delayed hypothermia in newborn piglets. Brain Research, 1154, 173-180. https://doi.org/10.1016/j.brainres.2007.03.083 
Lakatos, A., Kolossváry, M., Szabó, M., Jermendy, Á., Barta, H., Gyebnár, G., Rudas, G., \& Kozák, L.R. (2019). Neurodevelopmental effect of intracranial hemorrhage observed in hypoxic ischemic brain injury in hypothermia-treated asphyxiated neonates - an MRI study. BMC Pediatrics, 19(430), 1-11. https://doi.org/10.1186/s12887-019-1777-z

Madaschi, V. (2012). Tradução, adaptação transcultural e evidências de validade das Escalas Bayley III de Desenvolvimento Infantil em uma população do Município de Barueri, São Paulo [Dissertação de Mestrado, Universidade Presbiteriana Mackenzie]. Biblioteca Digital de Teses e Dissertações da Universidade Presbiteriana Mackenzie. http://tede.mackenzie.br/jspui/handle/tede/1591

Mastrangelo, M., Di Marzo, G., Chiarotti, F., Andreoli, C., Colajacomo, M. C., Ruggieri, A., \& Papoff, P. (2019). Early Post-cooling Brain Magnetic Resonance for the Prediction of Neurodevelopmental Outcome in Newborns with Hypoxic-Ischemic Encephalopathy. Journal of Pediatric Neurosciences, 14(4), 191-202. https://doi.org/10.4103/jpn.jpn_25_19

Mitra, S., Kendall, G. S., Bainbridge, A., Sokolska, M., Dinan, M., Uria-Avellanal, C., Price D., Mckinnon, K., Gunny, R., Huertas-Ceballos, A., Golay, X., \& Robertson, N. J. (2019). Proton magnetic resonance spectroscopy lactate/N-acetylaspartate within 2 weeks of birth accurately predicts 2 -year motor, cognitive and language outcomes in neonatal encephalopathy after therapeutic hypothermia. Archives of Disease in Childhood. Fetal and Neonatal Edition, 104(4), 424432. https://doi.org/10.1136/archdischild-2018-315478

Moher, D., Liberati, A., Tetzlaff, J., \& Altman. D. G. (2009). Preferred Reporting Items for Systematic Reviews and Meta-Analyses: The PRISMA Statement. PLoS Medicine, 6(7). https://doi.org/10.1371/journal.pmed.1000097

Montaldo, P., Lally, P. J., Oliveira, V., Swamy, R., Mendoza, J., Atreja, G., Kariholu, U., Shivamurthappa, V., Liow, N., Tei serskas, J., Pryce, R., Soe, A., Shankaran, S., \& Thayyil, S. (2019). Therapeutic hypothermia initiated within 6 hours of birth is associated with reduced brain injury on MR biomarkers in mild hypoxic-ischaemic encephalopathy: a non-randomised cohort study. Archives of Disease in Childhood. Fetal and Neonatal Edition, 104(5), 515-520. 10.1136/ archdischild-2018-316040

Montanha, S. U. S., Silva Filho, W. S., \& Frazão, D. W. P. (2020). A importância da ressonância magnética fetal no estudo de doenças do sistema nervoso central: revisão integrativa da literatura. Brazilian Journal of Development, 6(10), 74326-74344. https://doi.org/10.34117/bjdv6n10-019

Ouzzani, M., Hammady, H., Fedorowicz, Z., \& Elmagarmid, A. (2016). Rayyan-a web and mobile app for systematic reviews. Systematic Reviews, 5(210), 110. https://doi.org/10.1186/s13643-016-0384-4

Palisano, R., Rosenbaum, P., Walter, S., Russell, D., Wood, E., \& Galuppi, B. (1977). Development and reliability of a system to classify gross motor function in children with cerebral palsy. Development Medicine \& Child Neurology, 39(4), 214-223. https://doi.org/10.1111/j.1469-8749.1997.tb07414.x

Papazian, O. (2018). Encefalopatía Hipóxica-Isquémica Neonatal. Medicina (Buenos Aires), 78(Suppl 2), 36-41. http://www.medicinabuenosaires.com/PMID/30199363.pdf

Perez, J. M., Golombek, S. G., \& Sola, A. (2017) Clinical hypoxic-ischemic encephalopathy score of the Iberoamerican Society of Neonatology (Siben): A new proposal for diagnosis and management. Revista da Associação Médica Brasileira, 63(1), 64-69. https://doi.org/10.1590/1806-9282.63.01.64

Piper, M. C., \& Darrah, J. (1994). Motor assessment of the developing infant. Saunders.

Procianoy, R. S., \& Silveira, R. C. (2017). Encefalopatia Hipóxico-Isquêmica. In D. A. Burns, D. Campos Júnior, L. R. Silva, W. G. Borges, \& D. Blank (Org), Tratado de Pediatria: Sociedade Brasileira de Pediatria. (4a ed.), 2, 1677-1682. Manole.

Procianoy, R. S., Corso, A. L., Longo, M. G., Vedolin, L., \& Silveira, R. C. (2019). Therapeutic hypothermia for neonatal hypoxic-ischemic encephalopathy: magnetic resonance imaging findings and neurological outcomes in a Brazilian cohort. The Journal of Maternal-Fetal \& Neonatal Medicine, 32(16), 2727-2734. https://doi.org/10.1080/14767058.2018.1448773

Procianoy, R. S., Corso, A. L., Schoenardie, B. O., De Oliveira, G. P., Longo M. G., \& Silveira R. C. (2020). Outcome and Feasibility after 7 Years of Therapeutic Hypothermia in Southern Brazil. American Journal of Perinatology, 37(9), 955-961. https://doi.org/10.1055/s-0039-1692388

Rao, R., Trivedi, S., Distler, A., Liao, S., Vesoulis, Z., Smyser, C., \& Mathur, A. M. (2019). Neurodevelopmental Outcomes in Neonates with Mild Hypoxic Ischemic Encephalopathy Treated with Therapeutic Hypothermia. American Journal of Perinatology, 36(13), 1337-1343. https://doi.org/10.1055/s-00381676973

Rêgo, M. G. S., Vilela, M. B. R., \& De Oliveira, C. M. (2018). Óbitos perinatais evitáveis por intervenções do Sistema Único de Saúde do Brasil. Revista Gaúcha de Enfermagem, 39, e2017-0084. https://doi.org/10.1590/1983-1447.2018.2017-0084

Rodrigues, O. M. P. R. (2012). Escalas de desenvolvimento infantil e o uso com bebês. Educar em Revista, 43, 81-100. https://doi.org/10.1590/S010440602012000100007

Sarnat, H. B., \& Sarnat, M. S. (1976). Neonatal Encephalopathy Following Fetal Distress: A Clinical and Electroencephalographic Study. Archives of Neurology, 33, 696-705. https://doi.org/10.1001/archneur.1976.00500100030012 
Research, Society and Development, v. 10, n. 11, e356101119736, 2021

(CC BY 4.0) | ISSN 2525-3409 | DOI: http://dx.doi.org/10.33448/rsd-v10i11.19736

Silva, N. D. S. H., Lamy Filho, F., Gama, M. E. A., Lamy, Z. C., Pinheiro, A. L., \& Silva, D. N. (2011). Instrumentos de avaliação do desenvolvimento infantil de recém-nascidos prematuros. Revista Brasileira Crescimento Desenvolvimento Humano, 21(1), 85-98. http://pepsic.bvsalud.org/scielo.php?script=sci_arttext\&pid=S0104-12822011000100009

Sociedade Brasileira De Pediatria. (2019-2021). Hipotermia Terapêutica: Documento Científico (4a ed.). Departamento Científico de Neonatologia https://www.sbp.com.br/imprensa/detalhe/nid/hipotermia-terapeutica/\#: :text=A técnica de hipotermia vem,diagnóstico de encefalopatia hipóxico-isquêmica

Souza, R. G., Menezes, M. S. D., Castro, P. S., Carneiro, J. A., De Pinho, L., \& Caldeira, A. P. (2020). Atraso do desenvolvimento neuropsicomotor entre recémnascidos de alto risco acompanhados em um ambulatório de seguimento. Revista Norte Mineira de Enfermagem, 9(1), 57-66. https://doi.org/10.46551/rnm23173092202090107

Stillwell, S. B., Fineout-Overholt, E., Melnyk, B. M., \& Williamson, K. M. (2010). Evidence-based pratice, step by step: searching for the evidence. The American Journal of Nursing, 110(5), 41-47. https://doi.org/10.1097/01.naj.0000372071.24134.7e

Tagin, M. A., Woolcott, C. G., Vincer, M. J., Whyte, R. K., \& Stinson, D. A. (2012). Hypothermia for Neonatal Hypoxic Ischemic Encephalopathy: An Updated Systematic Review and Meta-analysis. Archives of Pediatrics \& Adolescent Medicine, 166(6), 558-566. https://doi.org/10.1001/archpediatrics.2011.1772

Takazono, P. S., \& Golin, M. O. (2013). Asfixia Perinatal: Repercussões Neurológicas e Detecção Precoce. Revista Neurociências, $21(1), 108-117$. https://doi.org/10.34024/rnc.2013.v21.8210

World Health Organization (2021). Number of neonatal deaths from birth asphyxia and birth trauma in the world. https://www.who.int/data/maternal-newbornchild-adolescent-ageing/indicator-explorer-new/mca/number-of-neonatal-deaths---by-cause

Zhong, G., Zhang, R., Jiaerken, Y., Yu, X., Zhou, Y., Liu, C., Lin, L., Tong, L. \& Lou, M. (2017). Better Correlation of Cognitive Function to White Matter Integrity than to Blood Supply in Subjects with Leukoaraiosis. Frontiers in Aging Neuroscience, 9(185), 1-7. https://doi.org/10.3389/fnagi.2017.00185 